\title{
Amlodipine poisoning revisited: Acidosis, acute kidney injury and acute respiratory distress syndrome
}

\author{
Kushal Naha, J. Suryanarayana, Riffat Abdul Aziz, Barkur Ananthakrishna Shastry
}

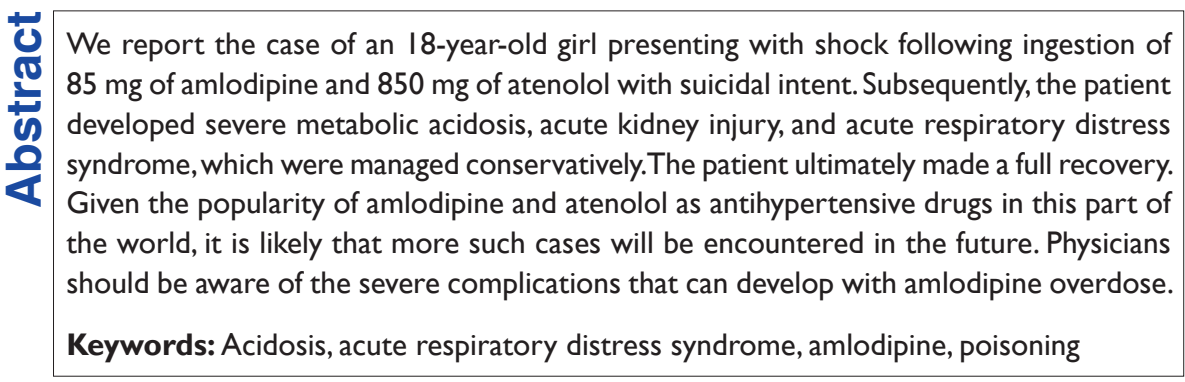

\section{Introduction}

Calcium channel blockers (CCB) constitute the leading form of cardiovascular drug overdose and have been implicated in up to $48 \%$ of deaths resulting from such overdose. ${ }^{[1]}$ Treating patients with overdose of CCBs can challenge even experienced physicians. The difficulty arises because patients severely poisoned with CCBs can develop profound refractory bradycardia, hypotension and either cardiogenic or noncardiogenic pulmonary edema. This case was reported to raise awareness about the severe complications of amlodipine poisoning, as well as the appropriate therapeutic approach to these complications among practicing medical professionals.

\section{Case Report}

The case we present here is about an 18-year-old girl who was referred to our hospital from a local health center with shock, following the consumption of 17 tablets containing a fixed dose combination of amlodipine $5 \mathrm{mg}$ and atenolol $50 \mathrm{mg}$. Gastric lavage had been performed at the local center prior to referral, approximately $2 \mathrm{~h}$

From:

Department of Medicine, Kasturba Hospital, Manipal, Karnataka, India

Correspondence:

Dr. Kushal Naha, Department of Medicine, Kasturba Hospital, Manipal - 576 104, Karnataka, India.

E-mail: kushalnaha@gmail.com

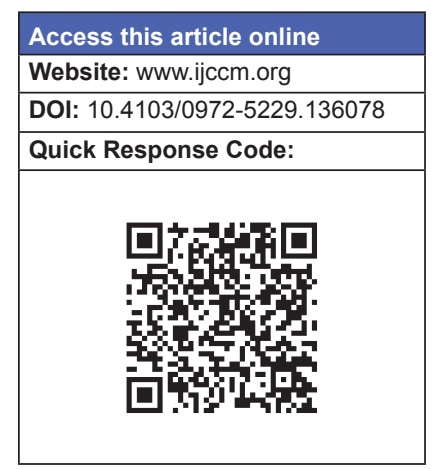

after the consumption of the tablets. She developed hypotension $2 \mathrm{~h}$ later and was transferred to our hospital $7 \mathrm{~h}$ after the time of ingestion. On questioning, she admitted to having ingested the tablets with suicidal intent. She denied concomitant consumption of alcohol or any other drugs.

At presentation, she was confirmed to be hypotensive, with cold peripheries, a thready pulse at $130 / \mathrm{min}$ and a blood pressure of $70 / 50 \mathrm{~mm} \mathrm{Hg}$ in the supine position. Respiratory rate was $26 / \mathrm{min}$. The patient was conscious and appeared anxious. Her Glasgow Coma scale was $15 / 15$. Systemic examination including cardiac and respiratory systems was entirely normal. Hourly urine output was initially below $0.5 \mathrm{ml} / \mathrm{kg}$ body weight but improved rapidly after infusion of intravenous (IV) fluids and vasopressors.

Pulse oximetry showed mild hypoxia $\left(\mathrm{spO}_{2}: 88 \%\right.$ on room air), which improved with oxygen supplementation. Routine laboratory tests showed mild renal failure (serum urea: $49 \mathrm{mg} / \mathrm{dl}$, creatinine: $1.9 \mathrm{mg} / \mathrm{dl}$ ). Other tests including complete blood counts, liver function tests and serum electrolytes were within the normal limits. Arterial blood gas analysis showed relative hypoxia with severe metabolic acidosis and respiratory compensation $(\mathrm{pH}: 7.31$, $\mathrm{pO}_{2}: 86 \mathrm{~mm} \mathrm{Hg}, \mathrm{pCO}_{2}: 12 \mathrm{~mm} \mathrm{Hg}, \mathrm{HCO}_{3}: 8.8 \mathrm{mmol} / \mathrm{l}$, $\left.\mathrm{FiO}_{2}: 0.6\right)$. Calculated parameters showed high anion gap 
metabolic acidosis (anion gap: 22), a $\mathrm{PaO}_{2} / \mathrm{FiO}_{2}$ ratio of 143 and an elevated A-a gradient (327 mm Hg). Serum lactate level was normal $(9.7 \mathrm{mg} / \mathrm{dl})$. Electrocardiography demonstrated sinus tachycardia with normal P-R and Q-T intervals. Chest $\mathrm{X}$-ray performed immediately after admission was normal.

The patient was immediately administered a $1 \mathrm{~L}$ bolus of IV normal saline. When hypotension failed to improve, inotropic support was initiated with an IV infusion of norepinephrine.

As hypoxia continued to worsen over the next $24 \mathrm{~h}$, chest X-ray was repeated and revealed bilateral extensive inhomogeneous shadows involving the middle and lower zones of both lung fields; both upper zones were spared [Figure 1]. Transthoracic echocardiography was performed, and cardiac dysfunction was excluded (ejection fraction $58 \%$, no evidence of diastolic dysfunction). Although direct measurement of pulmonary venous pressure could not be done, the overall picture was strongly suggestive of acute respiratory distress syndrome (ARDS).

Oxygen supplementation was provided initially through a venturi mask, and thereafter by means of non-invasive ventilation. IV calcium gluconate $(30 \mathrm{~mL}$ of $10 \%$ calcium gluconate IV stat followed by an infusion of $10 \%$ calcium gluconate at $10 \mathrm{~mL} / \mathrm{h}$ ) was administered when the patient developed ARDS.

The patient responded to inotropic support with progressive resolution of hypotension over the next $48 \mathrm{~h}$. ARDS also improved over the same period of time. Eight days after the episode, she had recovered completely

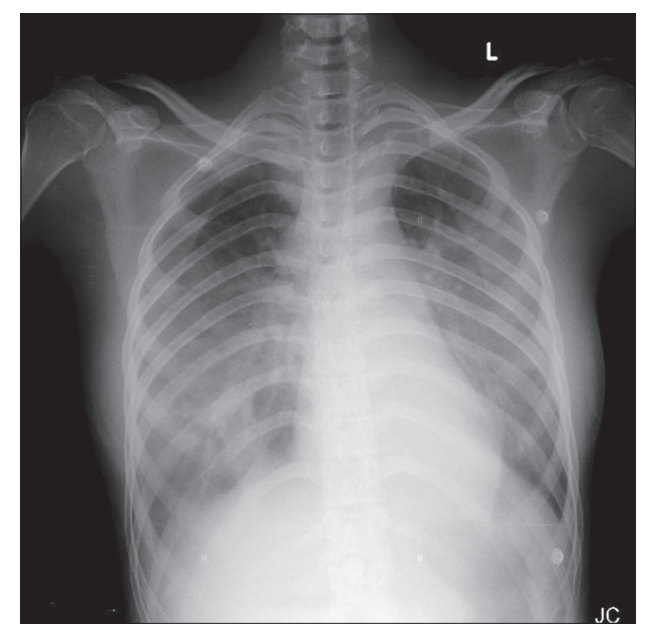

Figure I: Chest X-ray showing bilateral extensive inhomogeneous shadows involving the middle and lower zones of both lung fields, with sparing of both upper zones from hypotension, hypoxia and acute kidney injury. She was subsequently discharged after psychiatric evaluation and counseling.

\section{Discussion}

There are a limited number of case reports describing severe amlodipine intoxication; understandably therefore, there are no standardized guidelines for managing such cases. Gastrointestinal decontamination with activated charcoal constitutes the first line of therapy for patients with CCB overdose; ${ }^{[2]}$ unfortunately this approach is only beneficial when used within the $1^{\text {st }} \mathrm{h}$ of consumption, and could not be employed in our case. Other therapeutic strategies that have been used with varying degrees of success include the hyperinsulinemia-euglycemia technique ${ }^{[3]}$ continuous IV infusion of calcium, ${ }^{[4]}$ and inotropic support with dopamine, norepinephrine, dobutamine and levosimendan. ${ }^{[5]}$ In this case, it was decided to administer calcium infusion when the patient developed ARDS. This approach contrasts sharply with most case reports, wherein calcium infusion was employed to correct hemodynamic instability. For instance, Saravu and Balasubramanian described a young male with late onset ARDS after consuming $400 \mathrm{mg}$ of amlodipine, who received calcium gluconate for hypotension, prior to the onset of ARDS. ${ }^{[6]}$ Rizvi et al. described a female with refractory hypotension following consumption of $280 \mathrm{mg}$ of amlodipine, in whom treatment with calcium gluconate infusion yielded only marginal improvement in hypotension. ${ }^{[7]}$ Similarly Hasson et al. have reported another young female with refractory hypotension and ARDS after consuming $280 \mathrm{mg}$ of amlodipine..$^{[8]}$ Again, the patient was initiated on IV calcium gluconate primarily for the management of hypotension. Yet another case series by Ghosh and Sircar describes two individuals with refractory hypotension following amlodipine intoxication who had a successful outcome incorporating IV calcium infusion in their management. ${ }^{[4]}$ Kute et al. also described a patient with ARDS and hypotension who was administered IV calcium only when hypotension failed to respond to conventional inotropes. ${ }^{[9]}$ Selective pre-capillary vasodilation producing excessive pulmonary capillary transudation has been suggested as a mechanism for development of ARDS in patients with CCB overdose. ${ }^{[10]}$ In such a situation, administration of IV calcium raises extracellular calcium concentration, driving calcium intracellularly and thus reversing CCB induced vasodilation. Intuitively, such pharmacological antagonism would suggest that calcium gluconate be useful for primary management of CCB induced pulmonary edema. This hypothesis is consistent with the temporal patterns seen in this case, wherein progression of ARDS was seen in the first $24 \mathrm{~h}$ after 
admission despite correction of hypotension. However, further studies are required to confirm this hypothesis.

Another notable finding in this case was the development of severe metabolic acidosis, in the absence of lactic acidosis. This feature is also noticeable in most of the cases described above. We suggest that severe metabolic acidosis might be an independent sequel of amlodipine intoxication, with contribution from systemic hypotension and acute kidney injury.

Interestingly, this patient did not develop any overt signs of beta-blocker toxicity such as conduction blocks or even bradycardia despite the ingestion of $850 \mathrm{mg}$ of atenolol, instead presenting with reflex tachycardia probably secondary to amlodipine induced vasodilation and hypotension. It is possible that the relatively poor bioavailability of oral atenolol ${ }^{[11]}$ along with proven variability of biological response to high doses of atenolol could be responsible for the absence of beta-blocker toxicity in this instance. Pertinently, doses of up to $1200 \mathrm{mg} /$ day of atenolol have been reported to be well-tolerated without clinical manifestations. ${ }^{[12]}$

The relatively innocuous adverse effects of amlodipine such as peripheral edema and constipation, have resulted in a general perception that amlodipine is a safe and well-tolerated drug. While, this is certainly true of amlodipine in therapeutic doses, physicians should be aware of the severe organ dysfunction that can arise from overdosage of amlodipine given the popularity of this drug as an antihypertensive.

\section{References}

1. DeWitt CR, Waksman JC. Pharmacology, pathophysiology and management of calcium channel blocker and beta-blocker toxicity. Toxicol Rev 2004;23:223-38

2. Patel NP, Pugh ME, Goldberg S, Eiger G. Hyperinsulinemic euglycemia therapy for verapamil poisoning: A review. Am J Crit Care 2007; 16:498-503.

3. Azendour H, Belyamani L, Atmani M, Balkhi H, Haimeur C. Severe amlodipine intoxication treated by hyperinsulinemia euglycemia therapy. J Emerg Med 2010;38:33-5.

4. Ghosh S, Sircar M. Calcium channel blocker overdose: Experience with amlodipine. Indian J Crit Care Med 2008;12:190-3.

5. Varpula T, Rapola J, Sallisalmi M, Kurola J. Treatment of serious calcium channel blocker overdose with levosimendan, a calcium sensitizer. Anesth Analg 2009;108:790-2.

6. Saravu K, Balasubramanian R. Near-fatal amlodipine poisoning. J Assoc Physicians India 2004;52:156-7.

7. Rizvi I, Ahmad A, Gupta A, Zaman S. Life-threatening calcium channel blocker overdose and its management. BMJ Case Rep 2012;2012:1-4.

8. Hasson R, Mulcahy V, Tahir H. Amlodipine poisioning complicated with acute non-cardiogenic pulmonary oedema. BMJ Case Rep 2011;2011:1-3.

9. Kute VB, Shah PR, Goplani KR, Gumber MR, Vanikar AV, Trivedi HL. Successful treatment of refractory hypotension, noncardiogenic pulmonary edema and acute kidney injury after an overdose of amlodipine. Indian J Crit Care Med 2011;15:182-4

10. Humbert VH Jr, Munn NJ, Hawkins RF. Noneardiogenic pulmonary edema complicating massive diltiazem overdose. Chest 1991;99:258-9.

11. Keerthi H, Panakanti PK, Yamsani MR. Design and characterization of atenolol transdermal therapeutic systems: Enhancement of permeability via iontophoresis. PDA J Pharm Sci Technol 2012;66:318-32.

12. O'grady J, Anderson S, Pringle D. Successful treatment of severe atenolol overdose with calcium chloride. CJEM 2001;3:224-7.

How to cite this article: Naha K, Suryanarayana J, Aziz RA, Shastry BA. Amlodipine poisoning revisited: Acidosis, acute kidney injury and acute respiratory distress syndrome. Indian J Crit Care Med 2014;18:467-9.

Source of Support: Nil, Conflict of Interest: None declared. 